\title{
Genomic Similarity of Nucleotides in SARS CoronaVirus using K-Means Unsupervised Learning Algorithm
}

\author{
Jairaj Singh ${ }^{*}, 1$ \\ *Department of Computer Science and Engineering, *Birla Institute of Technology,Mesra
}

KEYWORDS

MachineLearning

K-Means

Sequences

Classification

\section{SUMMARY}

In addition to the guidelines provided in the abstract above,the following points summarizes the article below:

- The article discusses an application of Machine Learning in the field of virology.

- It aims to classify the SARS CoV2 virus as per the already known sequences of the bat-coronavirus, the Wuhan Sea Food Market pneumonia virus and the Wuhan coronavirus.

- To solve and predict the similarity of the SARS CoV2 coronavirus w.r.t other viruses discussed above,K-Means Unsupervised Learning Algorithm has been chosen.

- The data-set used is MN997409.1-4NY0T82X016-AlignmentHitTable.csv found on www.kaggle.com.(Complete link shared in the references section).[17]

Manuscript compiled: Monday $7^{\text {th }}$ September, 2020

${ }^{1}$ Corresponding author mail:mtity10005.19@bitmesra.ac.in
- The results have been validated by using a simple datacorrelation technique namely Spearman's Rank Correlation Coeffecient.

- I have also discussed my future work using Deep Neural Nets that can help predict new virus sequences and effectively find similarity if any with already discovered viruses.

\section{INTRODUCTION}

Corona-viruses are single-stranded positive-sense RNA viruses that are known to contain one of the largest viral genomes, up to around $32 \mathrm{kbp}$ (base pair) in length[1-5].In humans and birds, these viruses cause respiratory tract infections from mild range to lethal.With recent studies on the ever increasing genome sequences about the virus and the analysis made through prolonged research,the family of Coronaviridae can be classified into 4 distinct broad categories like the Alpha,Beta,Gamma and Delta versions of the corona-virus $[4,6,7,16]$. Alphacoronavirus and Betacoron- 
avirus affect mammalian hosts, those in Gammacoronavirus and the recently defined Deltacoronavirus mainly infect avian species. As per the recent phylogenetic studies, it is seen that the coronaviruses have had a long history of evolution and mutation with frequent zoonosis leading to cross-species infections.Many of the corona-virus strains are known to be of the bat origin leading to a vast pool of recombination and mutation opportunities leading to cross-species transmission affecting mammals and humans notably $[4,7,8,9,10,16]$. One of the most unique characteristics of the corona-virus is its highly long genome base-pair sequence which can adapt any kind of new information it picks up in the process of mutation which makes the classification even more challenging. The most lethal MERS and SARS corona-viruses belong to the sub-genus Sarbecovirus and the Marbecovirus of Betacoronavirus genera[9,11,12].Both of these result from the zoonotic transmission into humans leading to viral pneumonia,fever, breathing issues and other notable symptoms[14,15].From the analyses of the whole genome to viral protein-based comparisons, it was found that the COVID-19 virus belongs to the lineage B (Sarbecovirus) of Betacoronavirus.From phylogenetic analysis of the $R d-R p$ protein, spike proteins, and full genomes of the COVID-19 virus and other corona-viruses, it was found that the COVID-19 virus is most closely related to the bat-like corona-viruses[12,16-20].There is also ongoing debate that the whether the COVID-19 virus developed as a recombination with previously identified bat and unknown corona-viruses [16] or arose independently as a new lineage to infect humans[16].But with the identification of the $A C E-2$ receptor in humans and its notable affinity with the corona-virus spike protein it has become quite evident that the COVID-19 virus is none other than a derivative of the bat corona-virus. The following model here utilizes an alignment-based approach using the very famous K-Means Clustering algorithm which relies on various annotations of the viral genes like sequence length,number of basepairs present in the sequence and also the gaps or mutation affects on the information stored in the sequence.The benefits of using K-Means unsupervised learning algorithm lies in its easy of use in modelling complex data and high convergence rate resulting in accurate classifications.

\section{MATERIALS AND METHODS}

The experiment performed uses a Machine Learning approach to classify the sequence of the COVID-19 virus. I have used K-Means Unsupervised Learning algorithm primarily due to 2 factors:

- Due to the complexity of the data,comprising of various parameters which made it difficult to classify the virus into distinct labels.

- K-Means has a fast convergence rate and is easy to implement.

\section{Data Availability}

The data-set used in the implementation can be found on Kaggle(Google's ML platform)(Link for Data Used[17]).It is an accession-based data originally downloaded by performing a BLAST exhaustive search from NCBI(National Center for Biotechnology Information) database.The data has been described as per the following taxonomy.

- SARS-CoV-2(gene-bank)

- SARS-2019

- COVID-19 virus

- Wuhan corona virus

- Wuhan seafood market pneumonia virus.

Further,the columns in the table are further described as follows:
- MN997409.1 - - > SARS-CoV2-Accession Features

- MN997409.1.3 - - > Other SARS Accession Features

- $99.990-->$ percentage similarity between the virus strains.

- $29882-->$ the base pair alignment length of different virus strains.

- mismatches $-->$ mismatches in the sequence of SARS-CoV2 and other SARS accession features

- gap opens -- > open and closed gaps of the sequences, possibly due to mutation.

- q.start $-->$ the starting position of the SARS-CoV2 virus sequence

- q.end $-->$ the ending position of the SARS-CoV2 virus sequence

- s.start $-->$ the starting position of the other SARS-CoV2 virus sequence

- s.end $-->$ the ending position of the other SARS-CoV2 sequence. (see Figure 1)

\section{Statistical Analysis}

The implementation of K-Means algorithm is based on the logic to find sequences that belong to the same cluster.If the cluster is found to be same then it implies that those sequences have large number of identical parameters.Since the data does not have any well defined labels or classes, therefore it has to be trained without human supervision,i.e.using an unsupervised learning paradigm. The working of K-Means goes by the selection of centroids.Centroids are first allotted randomly and then by running for a certain number of iterations the K-Means algorithm fixes the centroids and allocates the clusters.

But the question is how does the algorithm know where to stop ?.For,that it uses a performance-metric called as inertia,which is the mean squared distance between each instance and its allocated centroid.

The algorithm runs so as to always minimize the inertia.The K-Means can be mathematically represented as $\sum_{1}^{k} \sum_{1}^{n}\left(\mathbf{x}_{i}-c_{j}\right)^{2}$.The $x_{i}$ and $c_{j}$ represent the instances and the allotted centroids respectively.There are two main kind of K-Means variants available namely random and batch.In random the centroids are allotted randomly and hence proceeds slowly whereas in batch K-Means the algorithm works much faster by avoiding unnecessary distance calculations.

\section{Optimal number of clusters}

In order to find the optimal number of clusters for a K-Means algorithm, it is recommended to choose either of the following four methods:

- Elbow method (which uses the within cluster sums of squares)

- Average silhouette method

- Gap statistic method

In this experiment, I have used Average silhouette method to decide the optimal number of centers on this data-set.The Silhouette method measures the quality of a clustering and determines how well each point lies within its cluster.The main advantage that this method has it gives a visual representation of the number of clusters optimized as per data that makes it easy to implement K-Means effectively. 
bioRxiv preprint doi: https://doi.org/10.1101/2020.10.12.336339; this version posted October 12, 2020. The copyright holder for this preprint (which was not certified by peer review) is the author/funder, who has granted bioRxiv a license to display the preprint in perpetuity. It is made available under aCC-BY-NC-ND 4.0 International license.

\begin{tabular}{|c|c|c|c|c|c|c|}
\hline A $\mathrm{MN} 99740 \ldots \equiv$ & A MN99740... $=$ & $\# 100.000 \equiv$ & \# $29882 \quad \equiv$ & $\# 0 \quad \equiv$ & \# 0 & \# 1 \\
\hline MN997409.1 & MT020881.1 & 99.990 & 29882 & 3 & 0 & 1 \\
\hline MN997409.1 & MT020880.1 & 99.990 & 29882 & 3 & 0 & 1 \\
\hline MN997409.1 & MN985325.1 & 99.990 & 29882 & 3 & 0 & 1 \\
\hline MN997409.1 & MN975262.1 & 99.990 & 29882 & 3 & 0 & 1 \\
\hline MN997409.1 & LC522974.1 & 99.993 & 29878 & 2 & 0 & 4 \\
\hline MN997409.1 & MT049951.1 & 99.987 & 29882 & 4 & 0 & 1 \\
\hline MN997409.1 & MT019532.1 & 99.987 & 29882 & 4 & 0 & 1 \\
\hline MN997409.1 & MN996528.1 & 99.987 & 29882 & 4 & 0 & 1 \\
\hline MN997409.1 & NC_045512.2 & 99.987 & 29882 & 4 & 0 & 1 \\
\hline
\end{tabular}

Figure 1 An overview of the data-table used in the experiment

\section{RESULTS AND DISCUSSION}

\section{Centroid Calculation}

$\mathrm{K}-\mathrm{Means}$ was implemented by exploiting the numeric values of the data such as the sequence length,percentage similarity ,open and closed gaps .Since the data has 10 columns in total, K-Means was given a random initialization of centroids. This made the algorithm cruise through all the values gradually and thereby predicting the best centroids found after convergence from 10 iterations. The metric got fixed at a value of $\mathbf{0 . 6 0 5 3 6}$ (see approximately hence proving that algorithm reached the point of convergence. (See Figure 2). For the experiment,only the families with at least 100 sequences were considered.This is done so that maximum information is covered while performing the clustering process.If there are less than 100 sequences,it implies that the sequences lost their genetic information due to improper mutation or other environmental conditions.

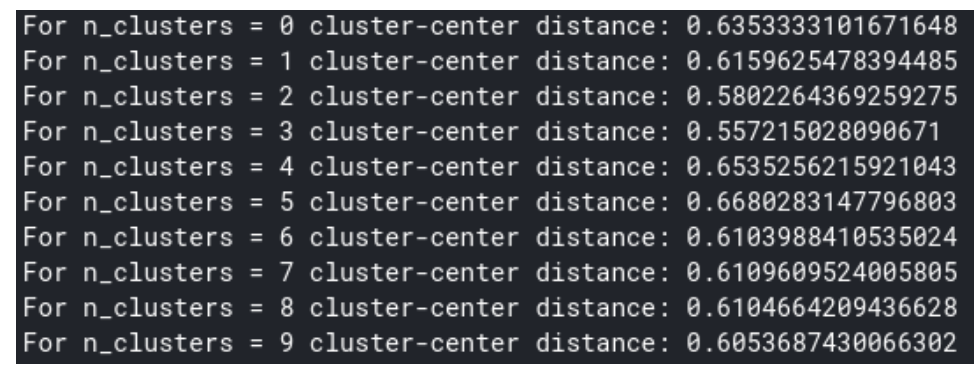

Figure 2 Computation of centroids through Silhouette method

\section{Cluster Formation}

The cluster formation in K-Means is achieved through 2 steps:

- By using Silhouette distance to compute the cluster-center distance.

- By assigning appropriate labels,so that classification is easy to visualize and understand.

The algorithm as seen from above discussion ran for 10 iterations forming the best cluster in the last iteration.
The K-Means algorithm can also be accelerated using the K-Means++ functionality in Python,where this model has been created. In the first iteration it can be seen that the clusters are oriented randomly as per the data, as the centroids have been randomly allotted.(see Figure 4- Cluster Formation)

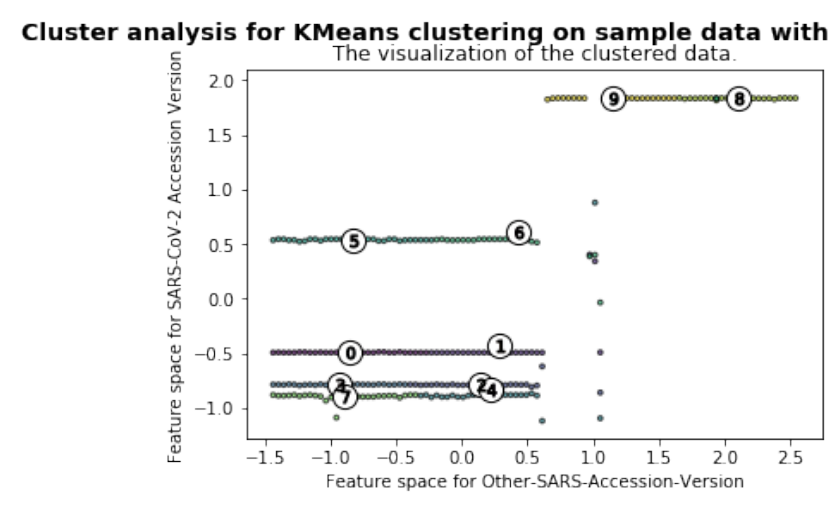

(a) 1st iteration of centroid allocation

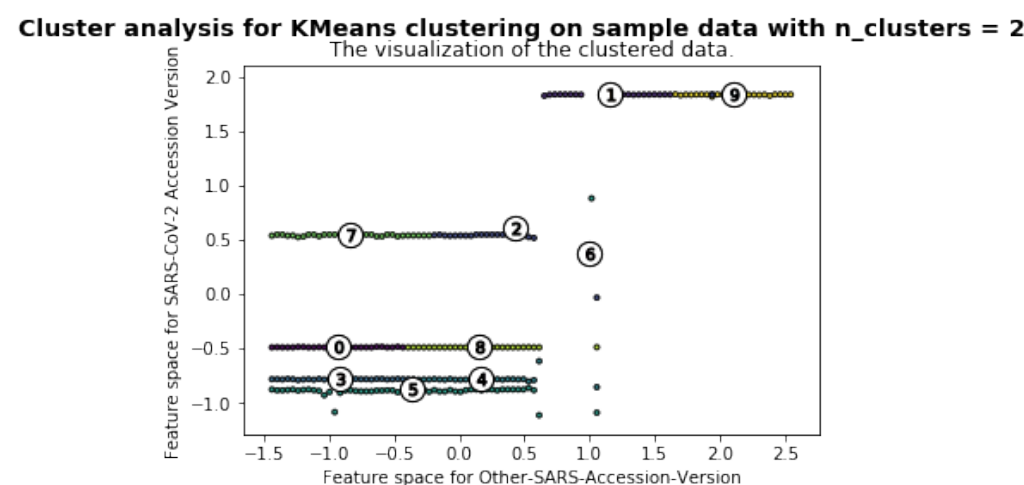

(b) 2nd iteration of centroid allocation

In the second iteration it can be seen that the centroids got calculated and the clusters start aligning themselves accurately and it continues in this way all the way up to the 10th iteration.Here only the first three iterations and then the final iteration of cluster formation are shown. 


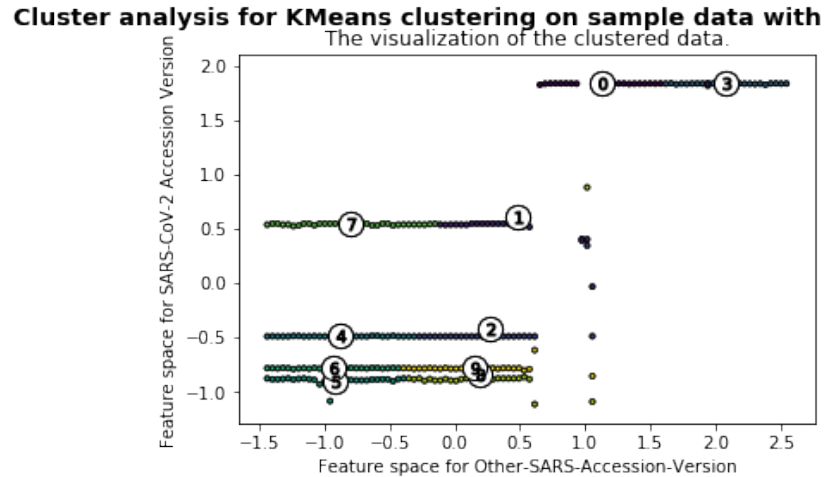

(a) 3rd iteration of centroid allocation
Thus we see that although the data-set was convoluted initially with no well defined labels, through this experiment we have been able to classify the virus reasonably into labelled-clusters.

\section{Validation}

The results here also confirms w.r.t the heat-map shown above in the results section, the closeness of the COVID-19 virus with the sequences from the Betacoronavirus genus by a quantitative analysis based on the Spearman's rank correlation used in the heat-map.(see Figure 5-Heat-Map)

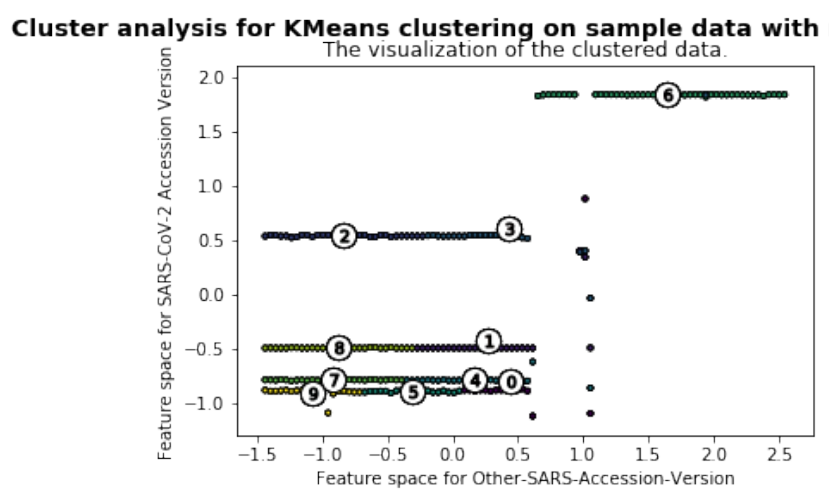

(b) 10th iteration of centroid allocation

Figure 4 : Cluster formation shown 1st,2nd,3rd and 10th iteration

Here the first three iterations and then the final 10th iteration are shown.What is to be noted here is that the clusters unlike in conventional methods are represented as circles,here it has been represented as dots. This has been done in order to output results the way a virus sequence appears naturally under a microscope to keep the visualization as natural as possible.

The diagram's $x$ - axis represents the scaled feature space of the bat corona-virus, the Wuhan corona-virus and the Wuhan seafood market pneumonia virus while the $y$-axis represent the current SARS-CoV-2 corona-virus feature space. Here the feature space is a scaled array of all instances described in the data.All these instances have numerical values and have been scaled within the range of -1.5 to 2.0.The scaling was automatically set by the program based on the minimum and maximum limits of the data used.

\section{Observation}

The observation of the results show that clusters with centres numbered 9,5 and 7,4 and 0 show a high correlation with each other and thus belong to the SARS-CoV-2 sequence.The cluster with cluster numbered 6 (as per the diagram above in the 10th iteration) does not belong to the SARS-CoV2-sequence as its instances are negatively correlated .

The other cluster centers that lie in between share some information w.r.t the current COVID-19 virus but cannot be classified completely as a SARS-CoV2 sequence.They have a mix of other corona-virus strains such as the Wuhan Sea Food Market Pneumonia virus and the Wuhan corona-virus.

More notably,centers visible on the same strains signify that those cluster of viruses share exactly the same features and hence belong to the same class of the virus.
Spear-man's Rank Correlation is a metric describing similarity and dissimilarity between a pair of two ranked variables associated with each other.It is better than Karl Pearson Correlation as it does not require well defined parameters to find relationship between data.Also it works well on categorical data which remain fixed(like age,length,mass) throughout the experiment. In our data-set the correlation is between the target SARS-CoV2 virus and the other SARS variants found in either pneumonia virus and the Wuhan Seafood Market Virus.It is a categorical data set because it has parameters such as length of bp,open-close-gaps,percentage similarity.

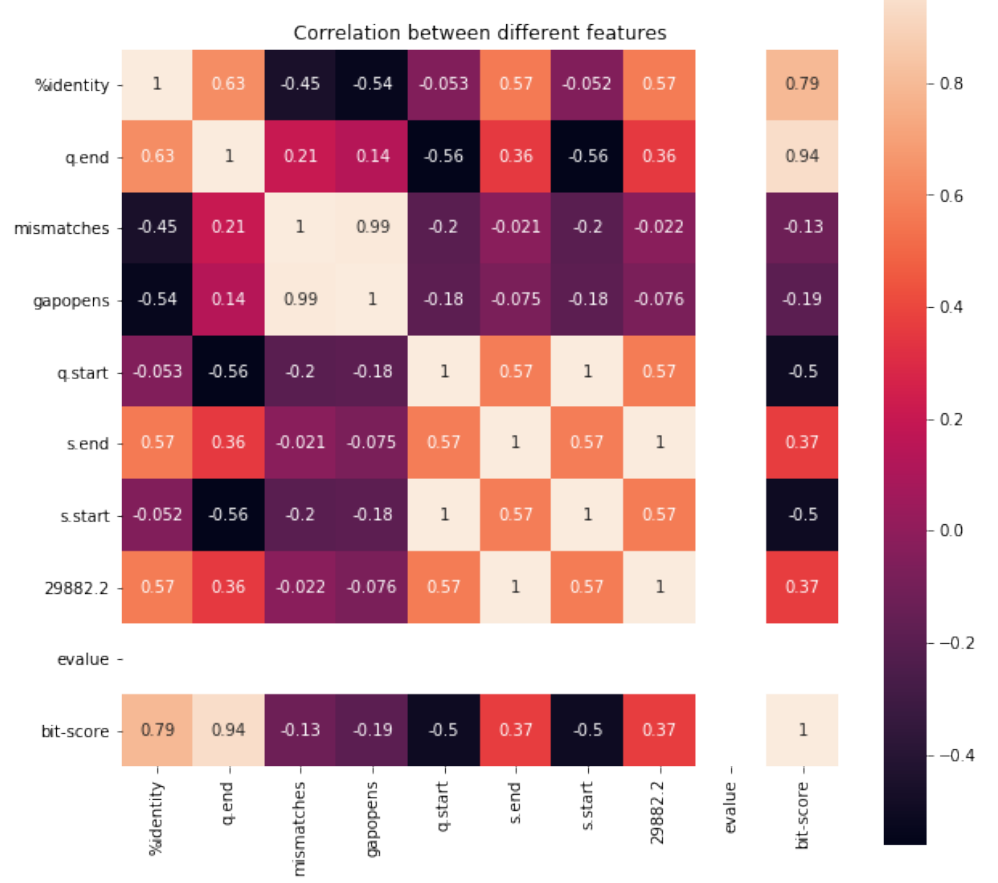

Figure 5 : Spear-man's Rank Correlation Heat-Map for result validation

Prior work elucidating the evolutionary history of the COVID19 virus had suggested an origin from bats prior to zoonotic transmission [12, 16]. Most early cases of individuals got infected with the COVID-19 virus originating in the Huanan South China Seafood Market [12-16]. This proves that Human-to-Human transmission is confirmed, further highlighting the need for continued 
intervention.Still, the early COVID-19 virus genomes that have been sequenced and uploaded are over 99 percent similar, suggesting these infections result from a recent cross-species event $[12,16]$.

\section{CONCLUSION}

Although the classification could be achieved through K-Means, the model also has the following limitations:

1.The data used is an alignment-based data which means that it would require tremendous computational resources (both space and time) to process even a larger data set with more data features.

2.Better classification can be achieved using a group of algorithms such as Linear SVM,Quadratic SVM and even a Polynomial SVM.[16]

Apart from these, even DBSCAN algorithm can be used as there is no need to specify the clusters.DBSCAN can automatically find the right shaped clusters from the data,no matter how complex. However,this model makes use of an alignment-based data for obtaining a suitable classification of SARS-CoV-2 nucleotide sequences. But the model can be further improved by incorporating even larger data-sets particularly with the use of multiple classification algorithms. One more method, which I am currently working on is using Deep Neural Network. The clear advantage DNN has that it is able to extrapolate high level features from low level ones.The second advantage is that DNNs can be trained in either of the three fundamental models of machine learning names Supervised Learning,Unsupervised Learning and Semisupervised learning.

DNNs can also be integrated quite easily with Natural Language Processing tools by using K-Mers algorithm(text based tokenizer) to extract the best sequence tokens based on an optimized length.(K=7 is the best in most cases). DNNs have a great role to play in the field of Artificial Intelligence as they hold the key to solve higher dimensional problems involving data complexity and providing diversified learning and higher efficiency. With the kind of AI we have today, computers would soon be able to help mankind achieve greater feats in the field of medical science.

\section{REFERENCES}

1.Weiss SR, Navas-Martin S. Coronavirus Pathogenesis and the Emerging Pathogen Severe Acute Respiratory Syndrome Coronavirus. Microbiol. Mol. Biol. 2005; Rev. 69: 635-664. https:/ /doi.org/10.1128/MMBR.69.4.635-664.2005.

2.Su S, Wong G, Shi W, Liu J, Lai ACK, Zhou J, et al. Epidemiology, Genetic Recombination, and Pathogenesis of Coronaviruses. Trends in Microbiology. 2016; 24: 490-502. https://doi.org/10.1016/j.tim. 2016.03.003 PMID: 27012512

3.Cui J, Li F, Shi ZL. Origin and evolution of pathogenic coronaviruses. Nature Reviews Microbiology. 2019; 17: 181-5192. https:/ /doi.org/10.1038/s41579-018-0118-9 PMID: 30531947

4.Schoeman D, Fielding BC. Coronavirus envelope protein: Current knowledge. Virology Journal. 2019;16. https:/ /doi.org/10.1186/s12985-019-1182-0 PMID:31133031 5.de Groot RJ, Baker SC, Baric R, Enjuanes L, Gorbalenya AE, Holmes KV, et al. Family Coronaviridae. In:King AMQ, Adams MJ, Carstens EB, Lefkowitz EJ, editors. Virus taxonomy. Ninth report of the international committee on taxonomy of viruses, Elsevier Academic Press; 2012. pp. 806-828.

6.Woo PCY, Lau SKP, Huang Y, Yuen KY. Coronavirus diversity, phylogeny and interspecies jumping.Experimental Biology and Medicine. 2009; 234: 1117-1127. https://doi.org/10.3181/0903MR-94 PMID: 19546349.

7.Wertheim JO, Chu DKW, Peiris JSM, Kosakovsky Pond SL, Poon LLM. A Case for the Ancient Origin of Coronaviruses. J. Virol. 2013; 87: 7039-7045. https://doi.org/10.1128/JVI.03273-12 PMID: 23596293

8.Luk HKH, Li X, Fung J, Lau SKP, Woo PCY. Molecular epidemiology, evolution and phylogeny of SARS coronavirus. Infection, Genetics and Evolution. 2019; 71: 21-30. https:/ / doi.org/10.1016/j.meegid.2019.03.001.

9.Vijaykrishna D, Smith GJD, Zhang JX, Peiris JSM, Chen H, Guan Y. Evolutionary Insights into the Ecology of Coronaviruses. J. Virol. 2007; 81: 4012-4020. https://doi.org/10.1128/JVI.0260506 PMID:17267506.

10.Drosten C, Günther S, Preiser W, van der Werf S, Brodt HR, Becker S, et al. Identification of a Novel Coronavirus in Patients with Severe Acute Respiratory Syndrome. N. Engl. J. Med. 2003; 348: 1967 - 1976.https:/ / doi.org/10.1056/NEJMoa030747 PMID: 12690091

11.Ksiazek TG, Erdman D, Goldsmith CS, Zaki SR, Peret T, Emery S, et al. A Novel Coronavirus Associated with Severe Acute Respiratory Syndrome. N. Engl. J. Med. 2003; 348: 1953-1966. https://doi.org/10.1056/NEJMoa030781 PMID: 12690092

13.Zaki AM, van Boheemen S, Bestebroer TM, Osterhaus ADME, Fouchier RAM. Isolation of a Novel Coronavirus from a Man with Pneumonia in Saudi Arabia. N. Engl. J. Med. 2012; 367: 1814-1820. https://doi.org/10.1056/NEJMoa1211721 PMID: 23075143

14.Guan Y, Zheng BJ, He YQ, Liu XL, Zhuang ZX, Cheung CL, et al. Isolation and characterization of viruses related to the SARS coronavirus from animals in Southern China. Science. 2003; 302: 276 278. https://doi.org/10.1126/science.1087139 PMID: 12958366

15.Alagaili AN, Briese T, Mishra N, Kapoor V, Sameroff SC, de Wit E, et al. Middle east respiratory syndrome coronavirus infection in dromedary camels in Saudi Arabia. MBio. 2014; 5. https:/ / doi.org/10. 1128/mBio.00884-14

16.Gurjit S. RandhawaID1, Maximillian P. M. SoltysiakID2, Hadi El RozID2 Camila P. E .Souza,Kathleen A. Hill2, Lila Kari Machine learning using intrinsic genomic signatures for rapid classification of novel pathogens: COVID-19 case study.https:/ /doi.org/10.1371/journal.pone.0232391 
bioRxiv preprint doi: https://doi.org/10.1101/2020.10.12.336339; this version posted October 12, 2020. The copyright holder for this preprint (which was not certified by peer review) is the author/funder, who has granted bioRxiv a license to display the preprint in perpetuity. It is made available under aCC-BY-NC-ND 4.0 International license.

17.Data-Set:https://www.kaggle.com/jamzing/sarscoronavirus-accession. 